\title{
DIAGNÓSTICO FOLIAR DE NUTRIMENTOS DE NUEVE ESPECIES FORESTALES TROPICALES DE TRES PISOS ALTITUDINALES EN CENTRO AMÉRICA
}

\author{
Alfredo Alvarado-Hernández ${ }^{1 / *}$, Ronald Chaves-Corea ${ }^{2}$, Eduardo Chacón-Madrigal ${ }^{3}$, \\ Wilberth Rodríguez-Alfaro ${ }^{4}$, Freddy Blanco-Montero ${ }^{5}$
}

Palabras clave: Concentración foliar; N-P-K-Ca-Mg-S-Fe-Mn-Zn-B-Cu-Al-Na; Alnus acuminata; Cedrela odorata; Cordia alliodora; Gmelina arborea; Tectona grandis; Terminalia amazonia; Vochysia guatemalensis; Vochysia ferruginea y Pachira quinata; Centroamérica.

Keywords: Foliar concentration; N-P-K-Ca-Mg-S-Fe-Mn-Zn-B-Cu-Al-Na; Alnus acuminata; Cedrela odorata; Cordia alliodora; Gmelina arborea; Tectona grandis; Terminalia amazonia; Vochysia guatemalensis; Vochysia ferruginea; Pachira quinata; Central America.

\section{RESUMEN}

Introducción. Es poca la información sobre concentración foliar de nutrimentos en especies forestales en Centro América. Se hace necesario desarrollar conocimiento que permita manejar de manera satisfactoria la nutrición de estos cultivos. Objetivo. Definir los niveles "normales" de concentración foliar al eliminar los valores atípicos fuera del rango de variación con un valor central entre el primer y tercer cuartil en histogramas de distribución de frecuencias de especies forestales. Materiales y métodos. Con material foliar de especies forestales de Costa Rica, Panamá, Guatemala, México y Colombia se confeccionó una base de datos con 3566 entradas con la que se preparó otra más pequeña para los análisis de las especies que se estudian en el presente documento, provenientes de 3 pisos

* Autor para correspondencia. Correo electrónico: alfredo.alvarado@ucr.ac.cr

1 Universidad de Costa Rica, Centro de Investigaciones Agronómicas, Facultad de Agronomía, San José, Costa Rica. (D) 0000-0002-6930-6660.

2 Universidad de Costa Rica, Escuela de Biología, Facultad de Ciencias, San José, Costa Rica. (D) 0000-0002-5003-0502.
ABSTRACT

Foliar nutrient diagnosis of nine tropical forestry species from three altitudinal belts of Central America. Introduction. Foliar nutrient concentration in forestry species of Central America is scarce. There is need to develop data to help in satisfactory handle nutrition of these crops. Objetive. To define "normal" foliar levels of nutrients considered as those that after eliminating atypical values in a histogram remain in the first and third quartiles of a normal frequency distribution of data available. Materials and methods. Foliar samples of forestry species collected in Costa Rica, Panamá, Guatemala, México and Colombia were analyzed and data were used to conform a data base with 3566 entries, a subset of data was prepared to generate the information

3 Universidad de Costa Rica, Centro de Investigaciones Agronómicas, Facultad de Agronomía, San José, Costa Rica.

(D) 00000-0002-8328-5456.

4 Universidad de Costa Rica, Centro de Investigaciones Agronómicas, Facultad de Agronomía, San José, Costa Rica.

(iD) 00000-0003-4516-8360.

5 Universidad de Costa Rica, Centro de Investigaciones Agronómicas, Facultad de Agronomía, San José, Costa Rica. (D) 00000-0002-6733-449X. 
altitudinales. Se calculó la mediana de valores de nutrimento para toda la población como base para determinar las desviaciones estándar y sus valores normales de concentración de N-P-K$\mathrm{Ca}-\mathrm{Mg}-\mathrm{S}-\mathrm{Fe}-\mathrm{Mn}-\mathrm{Zn}-\mathrm{B}-\mathrm{Cu}-\mathrm{Al}-\mathrm{Na}$. Resultados. Los resultados fueron una primera aproximación sobre la concentración foliar porcentual total en función del crecimiento de 9 especies forestales en los pisos altitudinales Tropical Húmedo-Muy Húmedo (4,23-6,77\%), Tropical Estacionalmente Seco (4,65-6,05\%) y Premontano (5,83-9,36\%). Son significativamente afectados por el piso altitudinal la concentración de $\mathrm{Al}$ en los pisos Tropical Húmedo-Muy Húmedo y el N en el Tropical Estacionalmente Seco. La distribución en porcentaje de las principales especies forestales estudiadas fue $C$. alliodora $(9,36), V$. guatemalensis $(6,77)$, C. odorata $(6,58)$, G. arborea $(6,05)$, P. quinata (5,91), A. acuminata (5,83), V. ferruginea $(4,81), T$. grandis $(4,65)$ y $T$. amazonia $(4,23)$. Conclusiones. En los pisos Tropical HúmedoMuy Húmedo la presencia de $V$. guatemalensis y $V$. ferruginea mostró elevados valores en mg. $\mathrm{L}^{-1}$ de $\mathrm{Al}(13470-22$ 172) $>\mathrm{B}(112-130)>\mathrm{Na}(401)$ y porcentuales de $\mathrm{P}(0,54-0,68)$. La especie $T$. amazonia presenta concentraciones porcentuales altas de $\mathrm{P}(0,59)>\mathrm{B}(164)>\mathrm{Cu}(46)$ en $\mathrm{mg} \cdot \mathrm{L}^{-1}$. En el bosque Tropical Estacionalmente Seco ninguna de las especies estudiadas (G. arborea, T. grandis y $P$. quinata) presentó concentraciones elevadas de ningún elemento de los estudiados. En el piso Premontano se encontró valores porcentuales elevados de $\mathrm{N}(3,57)>\mathrm{K}(1,13)$ en A acuminata, en $C$. alliodora de $\mathrm{N}(3,45)>\mathrm{Ca}(3,29)>\mathrm{K}(1,24)>$ $\mathrm{Mg}(1,02)$ y en $C$. odorata de $\mathrm{N}(3,22)$.

\section{INTRODUCCIÓN}

Uno de los principales problemas asociados al manejo de la nutrición de bosques y plantaciones forestales en regiones tropicales consiste en encontrar métodos de diagnóstico que faciliten for the 9 species considered in the present document obtained from 3 altitudinal belts. Analyses performed included N-P-K-Ca-Mg$\mathrm{S}-\mathrm{Fe}-\mathrm{Mn}-\mathrm{Zn}-\mathrm{B}-\mathrm{Cu}-\mathrm{Al}-\mathrm{Na}$. Results. Information presented is the first approximation about foliar total concentration of nutrients of nine forestry species in Central America growing in the Tropical Humid-Very Humid (4.23-6.77\%), Tropical Seasonally Dry (4.65-6.05\%) and Premontane (5.83-9.36\%) altitudinal belts. Foliar concentrations of Al in the Tropical HumidVery Humid and $\mathrm{N}$ in the Tropical Seasonally Dry were particularly large. The percentage of main forestry species analyzed was distributed as C. alliodora (9.36), V. guatemalensis (6.77), C. odorata (6.58), G. arborea (6.05), P. quinata (5.91), A. acuminata (5.83), V. ferruginea (4.81), T. grandis (4.65) and T. amazonia (4.23). Conclusions. The presence of $V$. guatemalensis and $V$. ferruginea in the Tropical Humid-Very Humid belts affected foliar concentrations to high values $\left(\mathrm{mg} . \mathrm{L}^{-1}\right)$ of $\mathrm{Al}(13$ 470-22 172)>B(112130) $>\mathrm{Na}(401)>\mathrm{P}(0.54-0.68 \%)$ and that of $T$. amazonia the concentrations of $\mathrm{P}(0.59 \%), \mathrm{B}(164$ $\left.\mathrm{mg} . \mathrm{L}^{-1}\right)$, and $\mathrm{Cu}\left(46 \mathrm{mg} . \mathrm{L}^{-1}\right)$. In the Tropical Seasonally Dry Tropical Forest none of the species studied (G. arborea, $T$. grandis and $P$. quinata) showed high foliar concentrations of any element. Foliar values found for $\mathrm{N}(3.57)>\mathrm{K}(1.13)$ in $A$. acuminata, of $\mathrm{N}(3.45)>\mathrm{Ca}(3.29)>\mathrm{K}(1.24)$ $>\mathrm{Mg}(1.02)$ in $C$. alliodora, and of $\mathrm{N}(3.22)$ in $C$. odorata in the Premontane belt were considered as high.

determinar cuáles son los factores nutricionales que en mayor medida limitan su productividad (Alvarado y Raigosa 2012). Según Lavender (1970), aunque el inicio del análisis químico de las plantas en 1804 se atribuye a Sausure al demostrar que el contenido de cenizas en los órganos de las 
plantas varía entre especies, su edad, órgano a analizar y el suelo sobre el que crecen, no es sino hasta en 1905 cuando Hall sugiere la utilización de esta práctica como guía para la toma de decisiones en la fertilización de cultivos.

Veinte años después se inicia la investigación para establecer estándares de comparación mejorando las tecnologías de análisis para estudiar el contenido de nutrimentos y se conforman 2 grupos de investigadores, los que preferían el análisis de muestras secas o el de material fresco para evitar pérdidas de algunos elementos por volatilización. El mismo Lavender (1970) cita que de las primeras décadas del siglo XX se conocen pocos estudios sobre el análisis de plantas, mencionando como sobresalientes los de Mitchell (1934) y Leyton (1948) como pioneros en la identificación de contenidos foliares y hasta de mantillo del suelo para establecer niveles de deficiencias, críticos y de consumo de lujo para algunas especies de coníferas de zonas templadas.

Aunque hoy en día el análisis foliar es un método de diagnóstico de requerimientos nutricionales bien establecido en agricultura y horticultura, su empleo es menos frecuente en el sector forestal donde se considera debe emplearse en combinación con otros procedimientos que permitan identificar el porqué de los niveles de insuficiencia y la manera de remediar el problema nutricional (Driessche 1974). Entre los métodos disponibles, el análisis de tejidos vegetales tiene relevancia al considerar en su empleo aspectos como los procedimientos de extracción de nutrimentos en el laboratorio, la definición del órgano (tejido) a analizar y la escogencia de los árboles a muestrear (Smith y Loneragan 1997).

El análisis foliar es un instrumento que permite determinar la concentración total de los nutrimentos en las acículas y hojas de los árboles de manera rápida y así comparar los valores obtenidos en diferentes partes del mundo por muchos investigadores (Binkley 1986, Drechsel y Zech 1991). Evans (1979) y Smith y Loneragan (1997) consideran que a pesar de las variaciones de la concentración foliar encontradas entre especies, es con esta información que se trata de establecer el estado nutricional de las plantaciones, para diagnosticar y predecir posibles trastornos de nutrimentos que se cuantifican para reponer los extraídos, en este caso por explotación de la madera. Varios autores (Drechsel y Zech 1993, Lugo et al. 2012, Alvarado 2015) mencionaron que los valores de concentración de los nutrimentos en el tejido foliar varían principalmente en función de la especie, las condiciones de sitio (p.e. la variación altitudinal), el manejo silvicultural y en menor grado por la edad de los sistemas forestales, la variación introducida durante el muestreo de los tejidos y la relacionada con el proceso de análisis a nivel de laboratorio (Corrales et al. 2005).

El efecto del componente genético es decir la especie, subespecie, variedad, procedencia, genotipo, clon o híbrido (Ballard 1984, Boardman y McGuire 1990, Drechsel y Zech 1991, Lehto et al. 2010) es muy utilizado en proyectos de mejoramiento genético donde se han observado diferencias en la concentración de ciertos elementos para distintas etapas de crecimiento, clase diamétrica (edad del árbol), desarrollo y posición del tejido foliar en la copa del árbol, la distribución de la biomasa de raíces en el suelo, la posición de las hojas en la copa, la caída del follaje, la autopoda, el estado fenológico del árbol y la edad del follaje (hojas jóvenes, maduras o viejas). En bosques inundables de la Amazonía Brasilera se ha encontrado que las hojas viejas son más ricas en $\mathrm{Ca}$ y $\mathrm{Mg}$ que las hojas jóvenes, que a la vez son más ricas en $\mathrm{K}$ y a menudo en $\mathrm{N}$, $\mathrm{P}$ y Na. Además, los contenidos foliares de las especies son más elevados en ecosistemas con suelos y ríos a su alrededor altos en $\mathrm{Ca}, \mathrm{Mg}$ y $\mathrm{Na}$ (Klinge et al.1983, Klinge 1985).

Desde el punto de vista altitudinal las especies comparadas del piso Premontano presentan las mayores concentraciones foliares acompañadas de una capacidad de absorción reducida, aspectos relevantes en proyectos de reforestación para conservar y mejorar el ambiente. Pregitzer y King (2005) indican que desde el punto de vista nutricional la capacidad 
de absorción de nutrimentos, decrece con la altura del sitio debido a (i) la reducción de la tasa de fotosíntesis causada por un aumento de la nubosidad y la baja radiación solar, (ii) la disminución de la temperatura y menores tasas de respiración y transpiración a mayor elevación y (iii) una menor disponibilidad de nutrimentos causada por la baja temperatura y bajos valores de $\mathrm{pH}$ que reducen la tasa de mineralización de residuos. Tanner et al. (1998) mencionan que la baja tasa de crecimiento de la vegetación en el Bosque Tropical Montano se debe a la reducida disponibilidad de nutrimentos y que a mayor altitud el contenido foliar de $\mathrm{N}$ disminuye, los de $\mathrm{P}$ y $\mathrm{K}$ a menudo disminuyen y los de Ca y $\mathrm{Mg}$ no presentan ninguna tendencia definida. Sin embargo, la información disponible sobre una reducción del contenido foliar de $\mathrm{P}$ en la montaña alta no es concluyente y más bien confirma la evidencia de bajos niveles de N (Kitayama y Aiba 2002, Soethe et al. 2008).

Por medio del análisis foliar se han identificado especies arbóreas y leñosas hipertolerantes o hiperacumuladoras de metales pesados (Baker y Brooks 1989, van der Ent et al. 2013, Leitenmaier y Küpper 2013), de importancia en proyectos de fitoremediación y como fitominadoras (Rascio y Navari-Izzo 2011). La definición de plantas hiperacumuladoras de uno o varios elementos se puede estudiar en los trabajos de varios autores quienes han descrito relaciones entre la disponibilidad de elementos en el suelo y los absorbidos por los árboles y especies o grupos de especies de plantas (Chernery y Sporke 1976, Haridasan 1982, Breulmann et al. 1996, Ma et al. 2005, Fernando et al. 2009).

Entre las condiciones de sitio que pueden afectar la concentración foliar de los nutrimentos se incluyen la pendiente y la elevación del terreno, la temperatura del sitio, la pedregosidad, la condición de drenaje, la profundidad y la fertilidad del suelo, el reciclaje de los nutrimentos, la variación y duración de la estacionalidad climática (diaria, anual e interanual) que define la época de muestreo (p.e. caída de follaje, floración, fructificación, etc.), la cantidad y distribución de la lluvia que adiciona y lava nutrimentos, la luminosidad y la frecuencia e intensidad de las quemas y la frecuencia de inundaciones (Alvarado 2015). Los factores mencionados a su vez influyen sobre el número de árboles a plantar por unidad de superficie, la variación natural entre los árboles individuales de la plantación, la poda, el raleo, el control de malezas, la sanidad del rodal, las quemas y la adición de enmiendas como fertilizantes y cal, la variación de producción de biomasa, la acumulación de nutrimentos y la producción y reciclaje de residuos en bosques nativos y plantaciones forestales.

Por la relevancia ambiental y la socioeconómica en términos de ingreso de recursos a las familias y empresas, de mantenimiento de ecosistemas y la recuperación de tierras degradadas, en el presente trabajo se consideró estudiar la dinámica de nutrimentos en el tejido foliar de especies de los pisos Tropical Estacionalmente Seco, Tropical Húmedo-Muy Húmedo y Premontano de Costa Rica, con 3 especies de cada piso altitudinal previamente descritas por Alvarado y Raigosa (2012). Se enfatiza en presentar la información existente sobre análisis foliares y su interpretación basándose en resultados obtenidos con muestras foliares de plantaciones y bosques naturales en Costa Rica, con la incorporación de otras de Colombia, Panamá, Guatemala y México según su disponibilidad. El principal punto de referencia para comparar los valores encontrados lo constituye el trabajo de Drechsel y Zech (1991) y a nivel centroamericano los de González (1996) y Alvarado et al. (1997).

\section{MATERIALES Y MÉTODOS}

La información utilizada en el presente trabajo proviene de estudios propios realizados en Costa Rica y Panamá y por otras personas autoras en Guatemala, México y Colombia para las especies Alnus acuminata (jaúl o aliso), Cedrela odorata (cedro amargo o spanishcedar), Cordia alliodora (laurel), Gmelina arborea (melina, yemane o gamar), Tectona grandis (teca o teak), Terminalia amazonia (roble coral 
o amarillón), Vochysia guatemalensis (cebo, mayo, chancho o yemeri), Vochysia ferruginea (botarrama, mayo colorado, chancho colorado) y Pachira quinata (pochote, ceiba roja o saqui-saqui). Basados en dicha información se confeccionó una base de datos con 3566 valores de concentración foliar, provenientes de 61 familias, 130 géneros y 203 especies.
Luego se calculó la mediana de valores para toda la población y nutrimento como base para determinar las desviaciones estándar y con ellas los valores normales de concentración en un subgrupo de muestras mayor a 18 de cada una de las 9 especies comparadas con resultados de análisis foliares para $\mathrm{N}, \mathrm{P}, \mathrm{K}, \mathrm{Ca}, \mathrm{Mg}, \mathrm{S}, \mathrm{Fe}$, $\mathrm{Mn}, \mathrm{Zn}, \mathrm{B}, \mathrm{Cu}, \mathrm{Al}$ y $\mathrm{Na}$ (Tabla 1 ). 


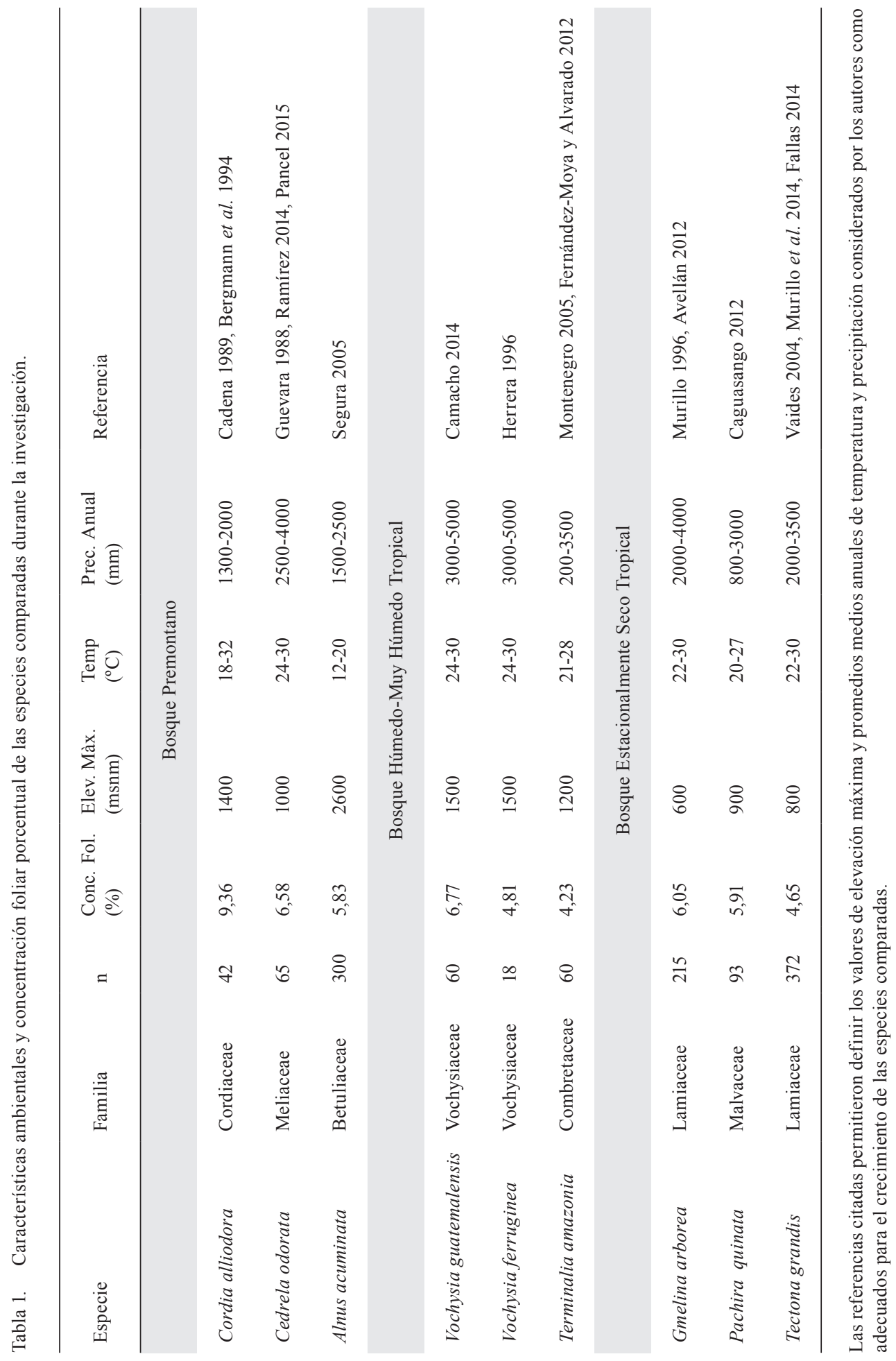


Metodología de muestreo. El tejido foliar analizado proviene de varios muestreos realizados durante la confección de trabajos de graduación de estudiantes de Licenciatura, Maestría y Doctorado en Costa Rica, Panamá, Guatemala, Colombia y México (p.e. Chaves-Corea 2021, Chacón-Madrigal et al. 2018). También incluye muestras tomadas en fincas y bosques del Trópico Húmedo, Muy Húmedo y Estacionalmente Seco por investigadores de la Universidad Nacional de Costa Rica en todo el país (Fonseca et al. 2016) durante 1999 y 2015. Las muestras provienen de sitios principalmente a menos de 2500 msnm con regímenes de temperatura isohipertérmicos y de humedad perúdico, údico y ústico. En los muestreos de siguió las normas descritas y recomendadas para cada especie previamente descritas por Alvarado et al. (1997).

Análisis de laboratorio. Las muestras de Costa Rica y Panamá se analizaron por $\mathrm{P}, \mathrm{Ca}$, $\mathrm{Mg}, \mathrm{K}, \mathrm{S}, \mathrm{Fe}, \mathrm{Mn}, \mathrm{Cu}, \mathrm{Zn}, \mathrm{B}, \mathrm{Na}$ y $\mathrm{Al}$ en los laboratorios de Suelos y Foliares del Centro de Investigaciones Agronómicas de la Universidad de Costa Rica en un aparato de espectrometría de emisión modelo ICP OES Perkin Elmer Optima 8300 con la metodología descrita por Kalra (1998) y la concentración de N mediante combustión seca en un analizador Elementar Rapid No 3 . Las muestras de otros países se analizaron por métodos similares descritos en los trabajos de los autores que permitieron su utilización.

Análisis de los datos. Se aplicó estadística descriptiva con los datos de la cantidad de individuos, medianas, desviaciones, errores estándar y rangos por elemento, asimismo, se calculó el valor intermedio por especie y por elemento. Para la estimación del rango de concentración foliar intermedio, se calculó el primer (25\%) y tercer cuartil $(75 \%)$ de cada elemento por especie, considerados como los valores mínimo y máximo del rango intermedio. Las especies forestales que se clasifican como acumuladoras de elementos son aquellas que poseen en la concentración foliar de uno o varios elementos y con las hiperacumuladoras se presentan de rojo en los Tablas 2, 3 y 4, medianas entre el percentil el $80 \%$ y el $95 \%$ de la población total. Aquellas especies en las que la mediana de la concentración foliar de uno o varios elementos se encuentra por arriba de la mediana global en más de 2 deviaciones estándar (mayor al percentil $95 \%$ ) se clasifican como hiperacumuladoras. Todas las especies cuya concentración foliar de elementos se encuentren entre los percentiles 25 y 80 se consideran como especies generalistas. En todos los casos los valores de todos los elementos se presentan en tablas, ordenados por especie y por concentraciones de mayor a menor, según algunos niveles internacionales considerados altos para la mayoría de las especies. 


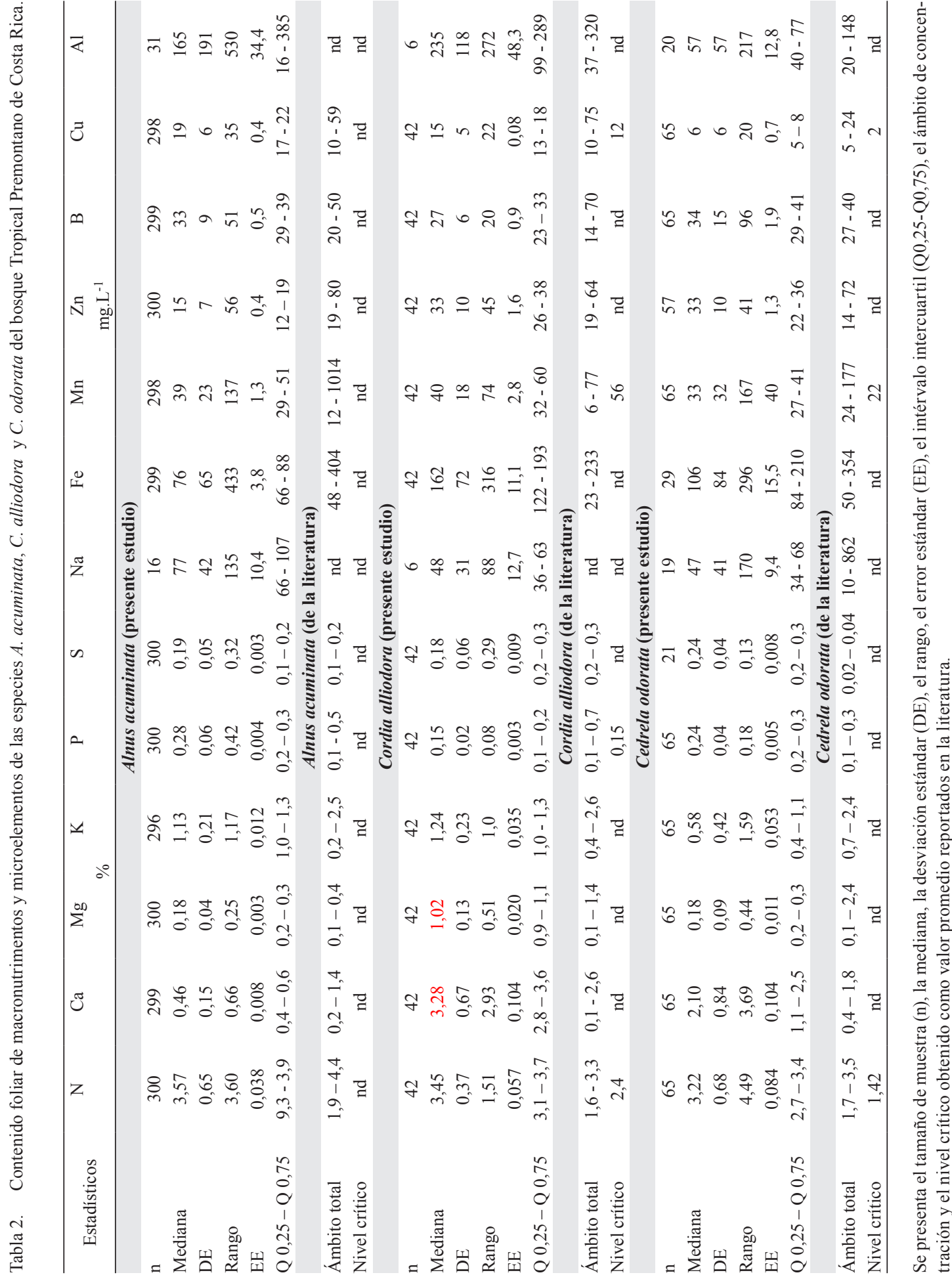




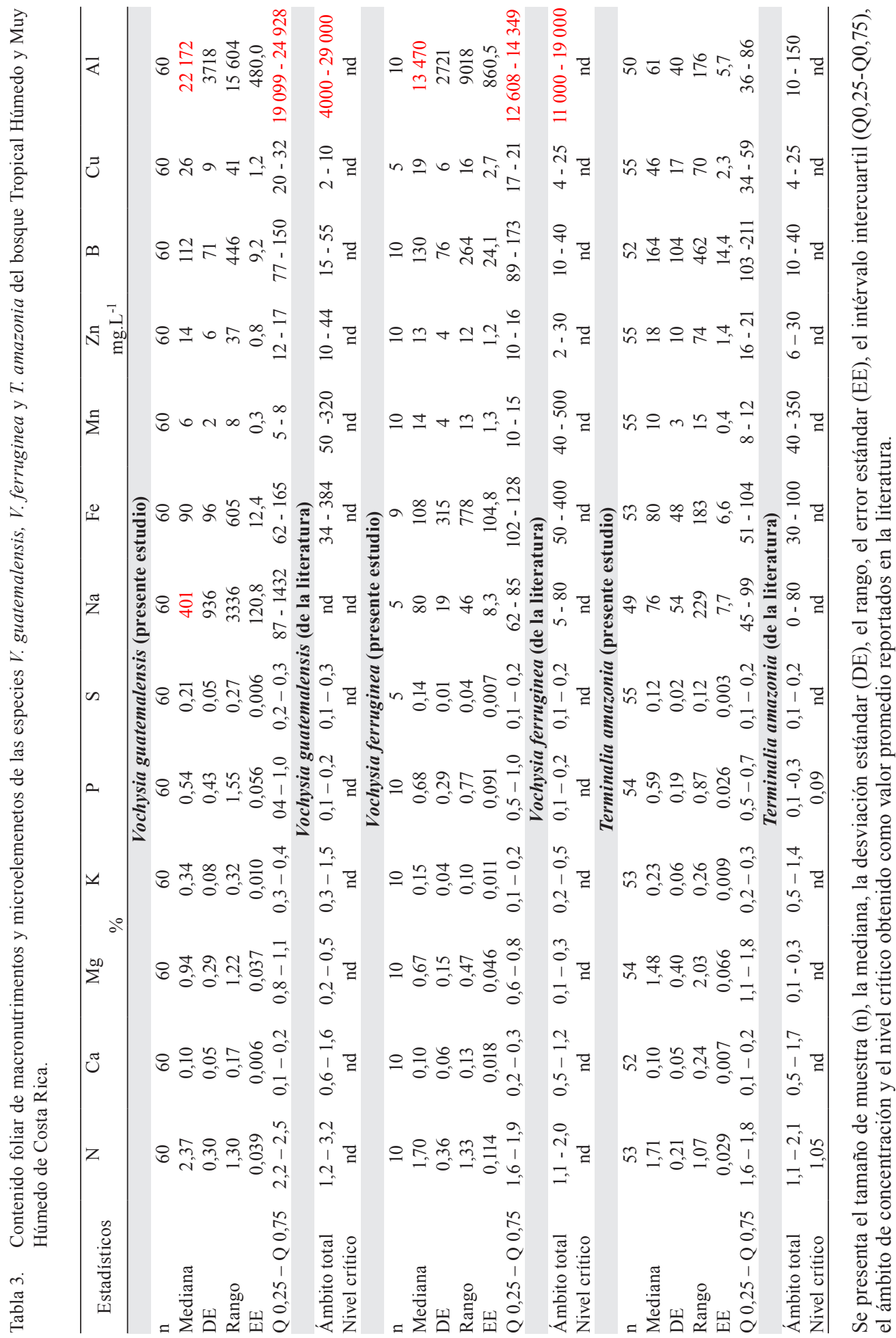




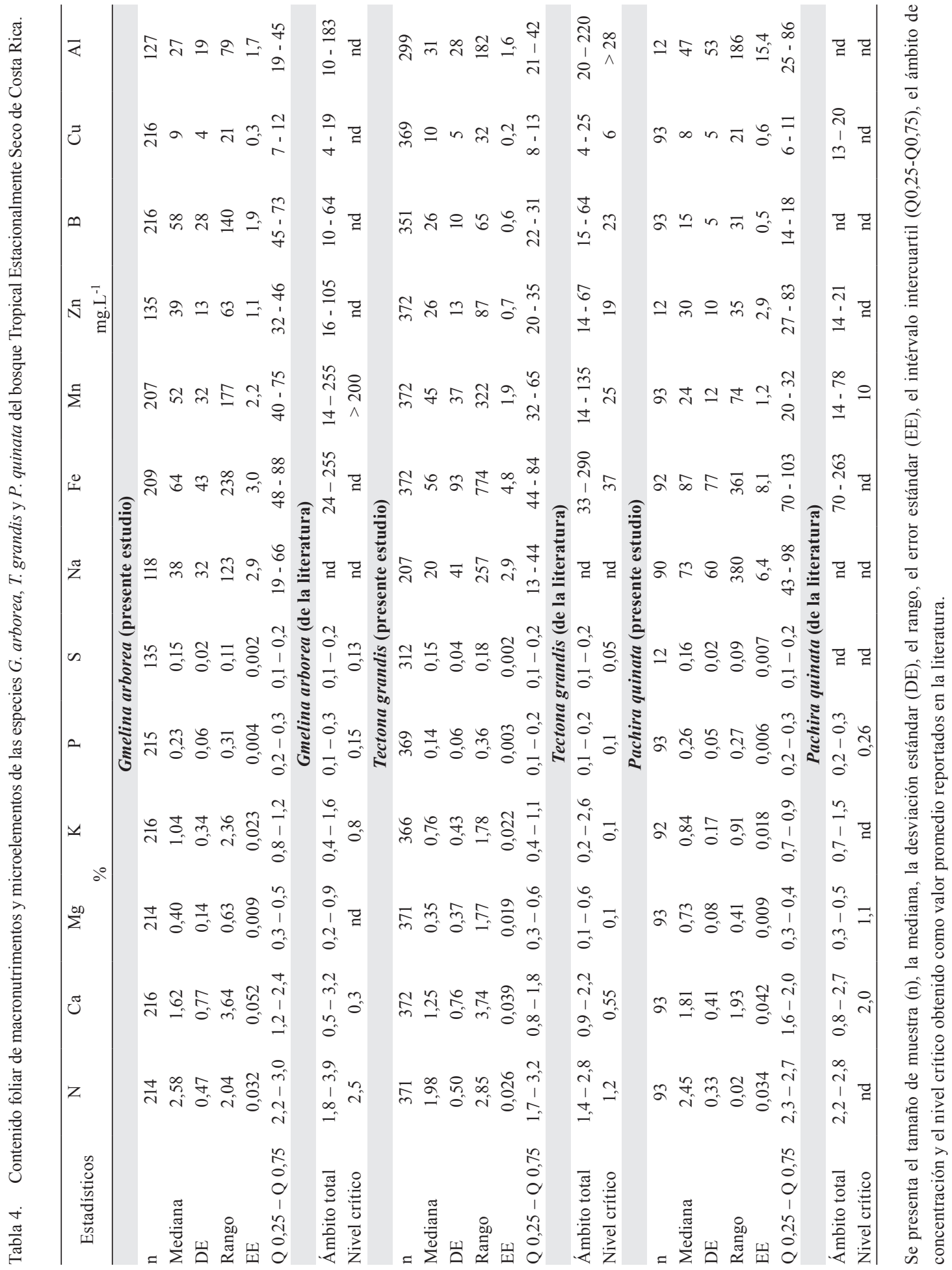


En general, se compararon los valores de concentración foliar de 9 especies recolectadas en el Trópico Americano para conocer su variación en función de la filogenia, ambiente de crecimiento y definir el rango de concentración normal de cada nutrimento y para cada especie. Se identificaron también, los valores que se consideran como acumulados e hiperacumulados y al final de cada especie se introdujeron 2 filas con rangos totales y niveles críticos de concentración foliar mencionados en la literatura. Las letras "nd" infieren que no existe información al respecto al alcance del personal del trabajo y por tanto los valores del presente estudio fueron nuevos para la ciencia.

\section{RESULTADOS Y DISCUSIÓN}

\section{Variación del contenido foliar en los ecosistemas comparados}

En la Tabla 1 se compara la concentración foliar porcentual (suma de todas las concentraciones porcentuales de los elementos analizados para cada especie) en función de los sitios estudiados. Este indicador muestra cómo los valores son propios para cada especie y sus rangos de variación por sitio son mayores en el piso Premontano $(5,83-9,36 \%)$ y menores pero similares entre sí en los pisos Tropical Húmedo y Muy Húmedo (4,23-6,77\%) y Tropical Estacionalmente $\operatorname{Seco}(4,65-6,05 \%)$.

En las especies del piso Estacionalmente Seco se encuentra la mayor concentración foliar de $\mathrm{N}$ y en los pisos Tropical Húmedo-Muy Húmedo se hiperacumulan los elementos $\mathrm{Al}$ y B. La baja concentración foliar de nutrimentos en las especies del piso Estacionalmente Seco es de gran importancia para la reforestación puesto que es aquí donde se plantan las especies de mayor relevancia económica en Costa Rica y en el resto del mundo. Al respecto, Raulino et al. (2020) mencionan que, en los Bosques Húmedos Tropicales de Pernambuco, Brasil las especies son más eficientes en la utilización de $\mathrm{N}$ y $\mathrm{Mg}$ mientras que en los Bosques Secos Tropicales las especies son más eficientes en el uso de P y K.

\section{Variación de la concentración foliar en el ecosistema Tropical Premontano}

En ese ecosistema domina la vegetación natural siempre verde con una cantidad moderada de epífitas y algunas especies deciduas durante la estación seca. El requerimiento altitudinal de cada especie (Tabla 1) puede ser diferente al del bioclima de muestreo, definido por varios autores (p.e. Bolaños y Watson 1993, Badilla et al. 2002, Pancel 2015) con un rango de precipitación anual entre 2000 y $4000 \mathrm{~mm}$, una biotemperatura que varía entre 17,5 y $24,0^{\circ} \mathrm{C}$ y un rango de elevación que oscila entre 250 y 1400 $\mathrm{m}$ en la vertiente Caribe y entre 700 y $1600 \mathrm{~m}$ en la vertiente pacífica de la América Central.

Alnus acuminata. Los valores de concentración foliar de la especie en el presente estudio disminuyeron en el orden (\%): $\mathrm{N}(3,57)>\mathrm{K}(1,13)$ $>\mathrm{Ca}(0,46)>\mathrm{P}(0,28)>\mathrm{S}(0,19) \approx \operatorname{Mg}(0,18)$ y $\left(\mathrm{mg} . \mathrm{L}^{-1}\right) \mathrm{Al}(165)>\mathrm{Na}(77) \approx \mathrm{Fe}(76)>\mathrm{Mn}(39)>$ $\mathrm{B}(33)>\mathrm{Cu}(19)>\mathrm{Zn}(15)$, considerándose altos los contenidos y los rangos de variación de $\mathrm{N}, \mathrm{P}$ y K y bajos los de Ca (Tabla 2). La información del presente trabajo coincide con la de Segura et al. (2006) en Andisoles de Costa Rica, pero es de mayor magnitud que la determinada para $A$. acuminata en Andisoles de Colombia (Escobar et al. 1993) y para otras especies del género Alnus que crecen en otros tipos de suelo (Rodríguez et al. 1984, Sharma 1993, Mills et al. 1996).

Rodríguez et al. (1984) y Cervantes y Rodríguez (1992) mencionan que los valores más elevados de N y P ocurren durante la época lluviosa, hecho atribuible a una mayor actividad en los nódulos de Frankia que aumentan la fijación $\mathrm{y}$ translocación de $\mathrm{N}$ y por asocio de $\mathrm{P}$ durante esta estación. Segura et al. (2006) mencionan que las concentraciones de $\mathrm{Mg}, \mathrm{Fe}, \mathrm{Zn}, \mathrm{Mn}, \mathrm{Cu}$ y $\mathrm{B}$ no disminuyen durante el año. Las concentraciones foliares de N, P y K concuerdan con las reportadas en la literatura para árboles del género Alnus (Rodríguez et al. 1984, Mills y 
Jones 1996, Sharma 1993, Segura et al. 2006) y las de Ca como bajas para 40 especies forestales Tropicales y SubTropicales (Drechsel y Zech 1991). Los valores de Mn se consideran bajos en relación con los de toxicidad de Mn (380-640 mg.kg-1) mencionados por Alvarado et al. (1997) y Alvarado y Raigosa (2012).

Cordia alliodora. Los valores de concentración foliar de la especie en el presente estudio disminuyen en el orden $(\%)$ : $\mathrm{N}(3,45)>\mathrm{Ca}(3,29)$ $>\mathrm{K}(1,24)>\operatorname{Mg}(1,02)>\mathrm{S}(0,18)>\mathrm{P}(0,15) \mathrm{y}$ $\left(\mathrm{mg} . \mathrm{L}^{-1}\right) \mathrm{Al}(235)>\mathrm{Fe}(162)>\mathrm{Na}(48)>\mathrm{Mn}(40)$ $>\mathrm{Zn}$ (33) $>\mathrm{B}$ (27) $>\mathrm{Cu}$ (15) (Tabla 2) donde se notan valores altos de $\mathrm{N}, \mathrm{Ca}, \mathrm{K}$ y $\mathrm{Mg}$ refleja el hecho de que la especie prefiere suelos de fertilidad natural media a alta. Al comparar los ámbitos de variación foliar hallados en el presente estudio con los valores de referencia internacional para la especie (Tabla 2) se nota que para el caso del K y el Al los valores presentan niveles adecuados, para los casos del N y Fe la mayoría pueden considerarse como adecuados y pocos como ligeramente bajos o ligeramente altos, los de $\mathrm{Ca}, \mathrm{Mg}, \mathrm{P}$ y $\mathrm{S}$ como ligeramente bajos y los de $\mathrm{Cu}, \mathrm{Zn}, \mathrm{B}$ y $\mathrm{Mn}$ como bajos en relación con lo encontrado por varios autores en plantaciones del Bosque Tropical Húmedo (Cadena 1989, Drechsel y Zech 1991, Zech y Drechsel 1992, Bergmann et al. 1994, Herrera y Finegan 1997, Davidson et al. 1998).

En las llanuras de la Zona Norte de Costa Rica, Bergmann et al. (1994) estudiaron la variación del contenido de la concentración foliar en 21 plantaciones de laurel con valores de árboles cloróticos menores que los encontrados en los árboles no cloróticos con concentraciones de $\mathrm{N}$ (2,4 vs $3,4 \%)$, P (0,15 vs $0,18 \%)$, Mn (56 vs 68 mg.kg-1) y $\mathrm{Cu}(12$ vs 20 mg.kg-1 $)$. En algunas situaciones la especie puede presentar valores foliares bajos causados por mal drenaje en las vegas de los ríos (Peck 1976) o en suelos en laderas degradadas abandonadas recientemente donde el sistema radical en suelos compactados por pastoreo es deficiente y la competencia de gramíneas es fuerte (Johnson y Morales 1972, Greaves y McCarter 1990).

Cedrela odorata. Los valores de concentración foliar de la especie en el presente estudio disminuyen en el orden $(\%): \mathrm{N}(3,22)>$ $\mathrm{Ca}(2,10)>\mathrm{K}(0,58)>\mathrm{P}(0,24)=\mathrm{S}(0,24)>\mathrm{Mg}$ $(0,18)$ y $\left(\mathrm{mg} \cdot \mathrm{L}^{-1}\right) \mathrm{Fe}(106)>\mathrm{Al}(57)>\mathrm{Na}(47), \mathrm{B}$ (34) $>\mathrm{Mn}$ (33) $=\mathrm{Zn}$ (33) $>\mathrm{Cu}$ (6) (Tabla 2). Los valores son comparables a los mencionados por otros autores en el Trópico Premontano (Zech y Drechsel 1992, Webb et al. 2000, Ramírez 2014). La concentración foliar de nutrimentos del cedro no varía significativamente con la edad de los árboles (Ramírez 2014) y según Zech y Drechsel (1992) y Webb et al. (2000) se consideran como deficientes los valores menores o iguales a $\mathrm{N}$ (1,42\%), Mn (22 mg.kg-1) y Cu (2 mg.kg $\left.{ }^{-1}\right)$.

\section{Variación de la concentración foliar en el ecosistema Tropical Húmedo a Muy Húmedo}

El Bosque Húmedo Pluvial se encuentra a una elevación media de 570 msnm (240-1200 $\mathrm{msnm})$, tiene una precipitación media anual de $2200 \mathrm{~mm}(1500-2400 \mathrm{~mm})$, la temperatura media anual es de $22^{\circ} \mathrm{C}\left(19-25^{\circ} \mathrm{C}\right)$ y el periodo efectivamente seco oscila entre 1-3 meses. El Bosque Muy Húmedo Tropical presenta un rango de precipitación promedio anual que va de los 4000 a los $5500 \mathrm{~mm}$, una temperatura promedio anual comprendida entre los $24^{\circ} \mathrm{C}$ y los $25^{\circ} \mathrm{C}$ y un periodo seco entre los 0-2 meses y se caracteriza por tener alta biodiversidad, especies siempreverdes con gambas, de alturas entre 40 a $50 \mathrm{~m}$, árboles altos y rectos, con troncos libres de ramas en los primeros 20 a $30 \mathrm{~m}$ y abundantes epífitas y lianas (información resumida de Bolaños y Watson 1993, Condit et al. 2011, Condit et al. 2013, Pancel 2015).

Vochysia guatemalensis. Los valores de concentración foliar de $V$. guatemalensis en el presente estudio disminuyen en el orden (\%): $\mathrm{N}$ $(2,37)>\operatorname{Mg}(0,94)>\mathrm{P}(0,54)>\mathrm{K}(0,34)>\mathrm{S}(0,21)$ $>\mathrm{Ca}(0,10)$ y $\left(\mathrm{mg}^{-L^{-1}}\right) \mathrm{Al}(22$ 172) $>\mathrm{Na}(401)>$ $\mathrm{B}(112)>\mathrm{Fe}(90)>\mathrm{Cu}(26)>\mathrm{Zn}(14)>\mathrm{Mn}(6)$ 
(Tabla 3). Los valores difieren a los encontrados previamente por otros investigadores (Pérez et al. 1993, González y Fisher 1997) quienes reportan concentraciones similares de $\mathrm{Ca}$ y $\mathrm{K}$ y menores de $\mathrm{B}$ con poca variación interanual en plantaciones entre 2 y 9 años de edad. La secuencia de concentración de los elementos menores presenta una tendencia a mantener niveles adecuados cómo mecanismo de adaptación a sitios degradados (Fisher 1995, Haggar et al. 1997, Carpenter et al. 2004). Los elevados valores de Al foliar permiten considerar el elemento como hiperacumulado y de acuerdo con Pérez et al. (1993) y González y Fisher (1997), por almacenar Al tanto en el follaje como en las raíces. Se consideran como bajos los contenidos foliares de $\mathrm{Ca}$ y altos los de N, P, Na y B mientras que los demás elementos concuerdan con los valores reportados en la literatura para la especie (Pérez et al. 1993, González y Fisher 1997, Montagnini 2000).

La variación del contenido foliar en la especie se atribuye a los cambios estacionales (Camacho 2014), su reciclaje de nutrimentos (Montagnini et al. 1993, Di Stéfano y Fournier 1998, Arias et al. 2011) y la variación causada por la procedencia genética (González y Fisher 1997). Camacho (2014) indica que la diferencia del contenido foliar en plantaciones de 2 a 21 años de edad causa un incremento de los contenidos foliares de $\mathrm{P}, \mathrm{Mg}$ y $\mathrm{Cu}$, que el contenido de $\mathrm{P}$ foliar es bajo y que se presentan interacciones entre sí y con otros nutrimentos entre los contenidos foliares de P, S, Zn y Al.

Vochysia ferruginea. Los valores de concentración foliar de esta especie disminuyen en el orden $(\%): \mathrm{N}(1,70)>\mathrm{P}(0,68) \approx \operatorname{Mg}(0,67)>$ $\mathrm{K}(0,15) \approx \mathrm{S}(0,14)>\mathrm{Ca}(0,10)$ y $\left(\mathrm{mg}^{\mathrm{L}} \mathrm{L}^{-1}\right) \mathrm{Al}(13$ $470)>\mathrm{B}(130)>\mathrm{Fe}(108)>\mathrm{Na}(80)>\mathrm{Cu}(19)>$ Mn (14) $\approx$ Zn (13) (Tabla 3) y no coinciden con la secuencia descrita por Herrera y Finegan (1997). La especie muestra valores de concentración foliar altos en $\mathrm{P}, \mathrm{Al}$ y $\mathrm{B}$ y bajos de $\mathrm{Ca}$, contrario a la idea de que la baja tasa de descomposición de los residuos en plantaciones indica que la concentración de N, P y K en el tejido foliar es baja
(Horn y Montagnini 1999). Reyes (1997) menciona de manera preliminar que altos contenidos de arcilla en posiciones cóncavas del relieve pueden causar anegamiento y afectar negativamente los contenidos foliares de $\mathrm{Zn}, \mathrm{P}$ y K. Como ha sido mencionado con anterioridad (Haridasan 1982, Geoghegan y Sprent 1996) la gran cantidad de Al absorbida por la especie no va en detrimento de las concentraciones foliares de otros elementos, por lo que se esperaba que la condición de tolerancia a la acidez del suelo se vea favorecida en el caso de que la fertilidad natural de los ecosistemas sea mayor.

Terminalia amazonia. Los valores de concentración foliar del amarillón disminuyen en el orden (\%): $\mathrm{N}(1,71)>\mathrm{Mg}(1,40)>\mathrm{P}(0,59)>$ $\mathrm{K}(0,23)>\mathrm{S}(0,12)>\mathrm{Ca}(0,10)$ y $\left(\mathrm{mg} . \mathrm{L}^{-1}\right) \mathrm{B}(164)$ $>\mathrm{Fe}(80)>\mathrm{Na}(76)>\mathrm{Al}(61)>\mathrm{Cu}(46)>\mathrm{Zn}(14)$ $>$ Mn (10) (Tabla 3). Se considera bajo el contenido foliar de $\mathrm{Ca}$ y elevados los de $\mathrm{P}, \mathrm{B}$ y $\mathrm{Cu}$, mientras que de acuerdo con Nichols et al. (1997) los valores de N, P y K se encuentran dentro del rango normal descrito para la especie y sobre los niveles críticos de N (1,05\%) y P (0,09\%) mencionados por los mismos autores. Montenegro (2005) indican que las concentraciones foliares de la especie aumentan durante la época lluviosa y las de $\mathrm{N}, \mathrm{K}, \mathrm{Ca}$ y $\mathrm{Mg}$ son mayores en plantaciones mixtas de 3 años que las encontradas en plantaciones puras de la misma edad. El efecto de sitio sobre la concentración foliar de $T$. amazonia es mínimo pues los valores no difieren mucho entre ecosistemas de fertilidad media a baja, francos a arcillosos en terrenos planos y ondulados (Montagnini 2000, Fernández-Moya y Alvarado 2012).

\section{Variación de la concentración foliar en el ecosistema Tropical Estacionalmente Seco}

El Bosque Tropical Estacionalmente Seco en Centroamérica está conformado por vegetación arbustiva, parches de vegetación sabanoide y cerca de los ríos de bosque caducifolio o siempreverde (Gómez 1986). Según varios autores (Murphy y Lugo 1995, Leiva 2007, Pancel 2015; 
Jiménez et al. 2015) presenta un régimen de temperatura media anual de $22^{\circ} \mathrm{C}\left(14-25^{\circ} \mathrm{C}\right)$, una elevación media de $750 \mathrm{msnm}$ (300-1950 $\mathrm{msnm}$ ) y se encuentra restringido por una precipitación media anual de $1400 \mathrm{~mm}$ (1000 a 2100 mm.año ${ }^{-1}$ ), un periodo seco de 3 a 6 meses de longitud con una alta variabilidad y distribución de la lluvia. Este ecosistema está sometido a quemas periódicas frecuentes en una región con una cantidad de lluvia anual que genera un déficit hídrico significativo a una elevación media de $750 \mathrm{msnm}$ (300-1950 msnm). Se lo encuentra sobre suelos neutros a ligeramente básicos y un periodo seco en el cual los árboles, particularmente leguminosas fijadoras de $\mathrm{N}$ tienden a perder el follaje y las raíces finas (Ordóñez 2003), de manera que durante la época de alta luminosidad no fotosintetizan debido a la limitante hídrica (Alvarado et al. 2018).

En la Tabla 4 se presenta la variación de la concentración foliar de las especies Gmelina arborea, Tectona grandis y Pachira quinata plantadas en el piso ecológico conocido como Bosque Tropical Estacionalmente Seco a una elevación inferior a $900 \mathrm{msnm}$ de la Costa Pacífica de América Central desde México hasta Panamá.

Gmelina arborea. Los resultados del presente estudio mostraron que la concentración foliar de nutrimentos en plantaciones de melina (Tabla 4) varían de mayor a menor en la secuencia $(\%) \mathrm{N}(2,58)>\mathrm{Ca}(1,62)>\mathrm{K}(1,04)>\mathrm{Mg}$ $(0,40)>\mathrm{P}(0,23)>\mathrm{S}(0,15)$ y $\left(\mathrm{mg} \cdot \mathrm{L}^{-1}\right) \mathrm{Fe}(65)>\mathrm{B}$ (58) $>\mathrm{Mn}(52)>\mathrm{Zn}(39) \approx \mathrm{Na}(38)>\mathrm{Al}(27)>\mathrm{Cu}$ (9), sin que ningún valor se considere como hiperacumulado. En general, los rangos se encuentran dentro de las concentraciones consideradas como adecuados para la especie por Murillo y Alvarado (2012), excepto los de S (0,15-0,16\%) que se consideran elevados y los de Al (38-68 mg. $\mathrm{L}^{-1}$ ) estimados como bajos según los mismos autores. En plantaciones en pendientes fuertes las concentraciones de Mn foliar tienden a ser más bajas que el valor de la mediana del presente trabajo (Stuhrmann et al. 1994) en suelos ácidos y erosionados, con baja disponibilidad de $\mathrm{N}$ y $\mathrm{P}$ en la Zona Norte de Costa Rica donde estos elementos limitan el desarrollo de la especie (Rojas y Murillo 2004).

Varios estudios de concentración foliar de nutrimentos en melina provienen de Centro América (Stuhrmann et al. 1994, González 1996, Murillo y Alvarado 2012, Avellán 2012) y muchos otros de zonas Tropicales fuera de la región (Drechsel y Zech 1991, Sampaio y Huber 1999, Paudyal 2012, Caguasango y Cadena 2012). En plantaciones adultas las mayores concentraciones de $\mathrm{K}$ se encuentran en la parte superior, las más altas de Ca en la parte media de la copa (Sampaio y Huber 1999) y Evans (1979) encontró que las concentraciones de N, P, Zn y $\mathrm{B}$ disminuyen y las concentraciones de $\mathrm{Ca}$ y $\mathrm{Fe}$ aumentan con el incremento de la sombra en la copa. En Costa Rica no se ha encontrado este efecto de copa, aunque si encontraron mayores concentraciones foliares de $\mathrm{N}, \mathrm{K}, \mathrm{Fe}$ y $\mathrm{Al}$ en la época lluviosa que en la época seca donde se obtuvo mayores concentraciones de $\mathrm{Ca}$ y $\mathrm{Mn}$ (Avellán 2012, Murillo y Alvarado 2012). De la información disponible, se concluye que los contenidos foliares de la especie varían con el tipo de suelo pues altos valores de saturación de Al inhiben la absorción de N y P, la intensidad lumínica del sitio, la edad fisiológica de las hojas, la posición de las hojas en la copa, la edad del árbol y la época de muestreo.

Varios investigadores (Drechsel y Zech 1991, Stuhrmann et al. 1994, Onweremadu 2007, Murillo y Alvarado 2012) han determinado niveles críticos de concentración foliar, sin embargo, la información aún no se considera suficiente pues no se ha correlacionado con ensayos de respuesta a la adición de los diferentes nutrimentos a nivel de campo. Stuhrmann et al. (1994) mencionan como valores críticos a nivel foliar concentraciones (\%) de $\mathrm{N}<2,50, \mathrm{P}<0,15, \mathrm{~S}<$ $0,13, \mathrm{~K}<0,80, \mathrm{Mn}>200 \mathrm{mg} . \mathrm{L}^{-1}$ y de la relación $\mathrm{K} / \mathrm{Mg}<2$ a lo que se añade el valor de $\mathrm{Ca}<0,30$ mencionado por Onweremadu (2007).

Tectona grandis. Los resultados del presente trabajo (Tabla 4) muestran que la 
concentración foliar de nutrimentos en plantaciones de teca varían de mayor a menor en la secuencia $(\%) \mathrm{N}(1,98)>\mathrm{Ca}(1,25)>\mathrm{K}(0,76)$ $>\operatorname{Mg}(0,35)>\mathrm{S}(0,15)>\mathrm{P}(0,14)$ y $\left(\mathrm{mg} \cdot \mathrm{L}^{-1}\right) \mathrm{Fe}$ (56) $>\mathrm{Mn}(45)>\mathrm{Al}(31)>\mathrm{B}(26)=\mathrm{Zn}(26)>$ $\mathrm{Na}(20)>\mathrm{Cu}(10)$, sin que ningún valor sea tan alto como para considerarlo hiperacumulado ni por debajo de los niveles críticos mencionados en la literatura (Nwoboshi 1975, Dreschel y Zech 1991, Montero 1999, Murillo et al. 2014). Sus rangos se encuentran dentro de los valores considerados como adecuados por Portuguez (2012) y Murillo (2013).

La variación del contenido foliar en teca se atribuye principalmente a efectos de la edad de la plantación, la época del año, la calidad del sitio y la densidad de muestreo (Drechsel y Zech 1991, Montero 1999, Siddiqui et al. 2009, Portuguez 2012, Fernández-Moya et al. 2013, FernándezMoya 2014). Se ha establecido que i) el N, P y K foliares decrecen durante los primeros años de crecimiento debido a reducciones de la productividad de las plantaciones con la edad, ii) que en las plantaciones más jóvenes aumentan las concentraciones foliares de $\mathrm{Ca}$ y $\mathrm{Mg}$ en función de la demanda fisiológica de los árboles más viejos y iii) que permanecen relativamente estables en el tiempo (Nwoboshi 1984, Negi et al. 1995, Kumar et al. 2009). Dreschel y Zech (1991) y Murillo et al. (2014) indican que el contenido foliar de Ca y $\mathrm{Mg}$ son mayores en sitios de crecimiento alto $\mathrm{y}$ que la teca crece bien aún a niveles de $\mathrm{Cu}$ en el suelo superior a los $200 \mathrm{mg}$. $\mathrm{L}^{-1}$. Fernández-Moya (2014) propone un umbral de $0,12 \%$ como nivel crítico de la concentración foliar de $\mathrm{P}$ en plantaciones de teca de América Central en base a un caso de estudio en plantaciones de teca en Panamá y revisión de literatura al respecto.

Pachira quinata. Se encontró que la mediana de concentración de macronutrimentos (Tabla 4) siguió el orden $(\%) \mathrm{N}(2,45)>\mathrm{Ca}(1,81)$ $>\mathrm{K}(0,84)>\operatorname{Mg}(0,37)>\mathrm{P}(0,25)>\mathrm{S}(0,16) \mathrm{y}$ los microelementos $\left(\mathrm{mg} . \mathrm{L}^{-1}\right) \mathrm{Fe}(87)>\mathrm{Na}(73)>$ $\mathrm{Al}(47)>\mathrm{Zn} \mathrm{(30)}>\mathrm{Mn}(24)>\mathrm{B}(15)>\mathrm{Cu}(8)$. Los valores normales encontrados en el presente estudio concuerdan bien con los rangos de variación mencionados para concentración foliar de los elementos estudiados por otros autores y los rangos de variación son normales excepto para $\mathrm{Na}, \mathrm{Fe}$ y Al que varían ampliamente y son nuevos a la literatura para S, Na, B y Al. La variación de la concentración foliar del pochote (Tabla 4) se compara con los valores adecuados, críticos $\mathrm{y}$ de deficiencia definidos por varios autores en Costa Rica (Drechsel y Zech 1991, Vázquez y Ugalde 1995, Vallejos 1996, Montero 1999) y por Caguasango (2017) en Colombia.

Montero (1999) demuestra que en plantaciones en el Pacífico Estacionalmente Seco de Costa Rica los contenidos foliares de K y $\mathrm{P}$ de pochote son 14 y $12 \%$ mayores en rodales entre 2 y 5 años y los de $\mathrm{Ca}$ y $\mathrm{Mg}$ superiores en un $40 \%$ a los encontrados en plantaciones de 8-25 años. Caguasango (2017) encontró una disminución no significativa de los contenidos foliares de $\mathrm{N}$ y $\mathrm{Mg}$ una tendencia altamente significativa de la concentración foliar de $\mathrm{Cu}\left(8-10 \mathrm{mg} . \mathrm{L}^{-1}\right)$ con la edad de los rodales y el índice de sitio y menor pero significativa con la concentración de $\mathrm{Zn}$ foliar. Al comparar los valores foliares encontrados con el crecimiento de las plantaciones en sitios de clases baja, media o alta Montero (1999) y Caguasango (2017) mencionan para concentraciones foliares "críticas" porcentuales de $\mathrm{Ca}<2,00$, de $\mathrm{K}<1,1$, de $\mathrm{P}<0,26$ y de $\mathrm{Zn}<10 \mathrm{mg} . \mathrm{L}^{-1}$, valores comunes en las plantaciones de Colombia de más de 15 años de edad. Montero (1999) encontró: i) un aumento de la concentración foliar de $\mathrm{Mg}$ conforme su contenido disponible en el suelo se eleva hasta $8 \mathrm{cmol}(+) \cdot \mathrm{L}^{-1}$, ii) que $0,50 \mathrm{cmol}(+) \cdot \mathrm{L}^{-1}$ $\mathrm{K}$ disponible en el suelo es suficiente para mantener su nivel foliar crítico sobre $1,1 \%$ y iii) un nivel calculado por modelaje superior a $10 \mathrm{mg}$ P.L ${ }^{-1}$ para lograr mantener en más de un $0,26 \%$ de $\mathrm{P}$ foliar. La concentración foliar de $P$. quinata en las plantaciones en el Bosque Seco Tropical de Bolívar, Colombia (Caguasango 2017) menciona valores deficientes de $\mathrm{Ca}(<2,00 \%)$ y de $\mathrm{Zn}(<$ $\left.10 \mathrm{mg} . \mathrm{L}^{-1}\right)$ en plantaciones de 17 a 24 años de edad. En relación con su posible deficiencia se considera que los valores normales del presente 
trabajo son adecuados para el crecimiento de la especie excepto los de $\mathrm{Ca}, \mathrm{K}$ y $\mathrm{Zn}$ que podrían considerarse como bajos.

\section{CONCLUSIONES}

La concentración foliar porcentual (suma de todos los elementos analizados para cada especie) muestra cómo los valores son propios para cada especie y sus rangos de variación por sitio son mayores en el piso Premontano (5,83-9,36\%) y menores pero similares entre sí en el Tropical Húmedo-Muy Húmedo (4,23$6,77 \%$ ) y en el Tropical Estacionalmente Seco $(4,65-6,05 \%)$.

Los rangos mencionados se ven significativamente afectados por la concentración foliar de los elementos mayoritarios como la concentración foliar de Al en el piso Tropical Húmedo-Muy Húmedo y la de N en el Estacionalmente Seco.

En orden descendente la concentración porcentual foliar total de las especies estudiadas fue C. alliodora (9,36), V. guatelamensis $(6,77)$, C. odorata (6,58), G. arborea (6,05), P. quinata $(5,91), A$. acuminata $(5,83), V$. ferruginea $(4,81)$, T. grandis $(4,65)$ y $T$. amazonia $(4,23)$.

Las 3 primeras especies mencionadas elevan su concentración foliar total debido a los aportes de N, Ca y Mg en C. alliodora y de Al, $\mathrm{Na}$ y B en $V$. guatemalensis y $V$. ferruginea.

Para varias de las especies estudiadas se generaron valores de concentración foliar considerados como nuevos a la literatura mundial, en particular de elementos menores.

En los pisos Tropical Húmedo-Muy Húmedo la presencia de $V$. guatemalensis y $V$. ferruginea mostró elevados valores en $\mathrm{mg} . \mathrm{L}^{-1} \mathrm{de}$ $\mathrm{Al}(13470$ - 22 172) > Na (401) > B (112-130) y además concentraciones porcentuales elevadas de $\mathrm{P}(0,54-0,68)$. La especie $T$. amazonia también presenta concentraciones porcentuales altas de $\mathrm{P}$ $(0,59)$, en mg.L.-1 de B (164) y de Cu (46).

En el Bosque Tropical Estacionalmente Seco ninguna de las especies estudiadas $(G$. arborea, T. grandis y $P$. quinata) presentó concentraciones elevadas de ningún elemento de los estudiados.

En el piso Premontano se encontró valores porcentuales elevados de $\mathrm{N}(3,57)>\mathrm{K}(1,13)$ en $A$. acuminata, de $\mathrm{N}(3,45)>\mathrm{Ca}(3,29)>\mathrm{K}$ $(1,24)>\operatorname{Mg}(1,02)$ en C. alliodora y de $\mathrm{N}(3,22)$ en $C$. odorata.

\section{AGRADECIMIENTOS}

Varias personas y empresas colaboraron desinteresadamente en el proceso de colectar el material vegetal del presente trabajo en sus bosques y plantaciones a los cuales les quedamos imperecederamente agradecidos: Ricardo Luján y Felipe Hidalgo de la empresa Barca SA, Ernesto Castillo datos de su tesis de Maestro en Ciencias en Tabasco, Méjico, Alvaro Castillo de la Compañía Nacional de Fuerza y Luz, Folker Kottman y Vinicio Ríos de la empresa Panamerican Woods, José E. Villalobos y Jaime Raigosa de la empresa privada, Edwin Estuardo Vaides datos de su tesis de Magister Scientae en Guatemala, Luis Salazar de CoopeAgri RL y Carlos L. Sandí y Rolando Camacho en terrenos de la EARTH y sus alrededores. Al procurar información pertinente al tema, fue invaluable la ayuda de Susana Aguilar y Gilbert Fuentes encargados de la unidad de documentación de la OET quienes permitieron ubicar pocas publicaciones de mucho valor científico desde 1934 y la gran mayoría de ellas a partir de 1990. Se agradece al Dr. Raúl Jaramillo y la Mg. Sc. Floria Bertsch por su colaboración institucional en el financiamiento parcial del trabajo.

\section{LITERATURA CITADA}

Alvarado, A. 2015. Plant nutrition in tropical forestry. In Pancel, L; Köhl, M (eds.). Tropical Forestry Handbook, 2. Springer-Verlag, Berlin, Heidelberg. p. 1113-1202.

Alvarado, A; Herrera, B; Orlich, T. 1997. Variación de las concentraciones foliares de especies utilizadas en proyectos de reforestación en Costa Rica. In Tercer Congreso Forestal Nacional: Unidos por el Desarrollo del Recurso Forestal Ante el Próximo 
Milenio. Resúmenes de ponencias. San José, Costa Rica. p. 302-304.

Alvarado, A; Mora, A; Chacón-Madrigal, E; Villalobos, JE; Sandí, CL. 2018. Concentración foliar de macro-y micro-nutrimentos en cuatro leguminosas maderables del trópico Estacionalmente Seco de Costa Rica. Revista Biología Tropical 66(3):969-983.

Alvarado, A; Raigosa, J. 2012. Nutrición y fertilización forestal en regiones tropicales. San José, Costa Rica. Asociación Costarricense de la Ciencia del Suelo. 411 p.

Arias, D; Calvo-Alvarado, J; Richter, DDB; Dohrenbusch, A. 2011. Productivity, aboveground biomass, nutrient uptake and carbon content in fast-growing tree plantations of native and introduced species in the Southern Region of Costa Rica. Biomass and Bioenergy 35(5):1779-1788.

Avellán, MJ. 2012. Determinación y variación de la concentración foliar de nutrimentos de Gmelina arborea Roxb. en Osa, Golfito y Corredores, Costa Rica. Tesis Lic. Heredia, Costa Rica, Universidad Nacional. $87 \mathrm{p}$.

Badilla, Y; Murillo, O; Obando, G. 2002. Efecto de la zona de vida y la altitud en la mortalidad y adaptabilidad al primer año de especies forestales en la Cordillera Volcánica Central, Costa Rica. Agronomía Costarricense 26(1):7-15.

Baker, AJM; Brooks, R. 1989. Terrestrial higher plants which hyperaccumulate metallic elements. A review of their distribution, ecology and phytochemistry. Biorecovery 1(2):81-126.

Ballard, R. 1984. Fertilization of plantations. In Bowen, GD; Nambiar, KS (eds.). Nutrition of Plantation Forests. San Diego, California, USA, Academic Press. p. 327-360.

Bergmann, C; Stuhrmann, M; Zech, W. 1994. Site factors, foliar nutrient levels and growth of Cordia alliodora plantations in the humid lowlands of Northern Costa Rica. Plant and Soil 166(2):193-202.

Binkley, D. 1986. Forest nutrition management. New York, USA, John Wiley \& Sons. 290 p.

Boardman, R; McGuire, DO. 1990. The role of zinc in forestry. I. Zinc in forest environments, ecosystems and tree nutrition. Forest Ecology and Management 37(1-3):167-205.

Bolaños, RA; Watson, V. 1993. Mapa ecológico de Costa Rica: según el sistema de clasificación de zonas de vida del mundo de LR Holdridge. San José, Costa Rica, Centro Científico Tropical. s.p.

Breulmann, G; Ninomiya, I; Ogino, K. 1996. Distribution characteristics of mineral elements in tree leaves of a mixed dipterocarp forest in Sarawak, Malaysia. Tropics 6(1/2):29-38.

Cadena, ME. 1989. Análisis nutricional de la especie Cordia alliodora asociado a hidroponía. Colombia Forestal 3(5):6-16.
Caguasango, SM. 2017. Predicción de rendimientos para plantaciones de Acacia mangium Willd. en la altillanura plana a partir de variables biofísicas Tesis M.Sc. Bogotá, Colombia, Universidad Nacional de Colombia. 96 p.

Caguasango, SM; Cadena, ME. 2012. Modelación de la calidad de índice de sitio-suelo sitio específico mediante criterios de disponibilidad de nutrientes en plantaciones de Gmelina arborea y Pachira quinata en bosque seco tropical (Zambrano-Bolívar). Tesis Lic. Bogotá, Colombia, Universidad Distrital Francisco José de Caldas. 230 p.

Camacho, M. 2014. Modelos de absorción de nutrimentos como herramientas para hacer recomendaciones de manejo en plantaciones de Vochysia guatemalensis Donn. Smith en el Trópico Muy Húmedo de Costa Rica. Tesis Lic. San José, Costa Rica, Universidad de Costa Rica. 103 p.

Carpenter, FL; Nichols, JD; Sandi, E. 2004. Early growth of native and exotic trees planted on degraded tropical pasture. Forest Ecology and Management 196(23):367-378.

Cervantes, E; Rodríguez, C. 1992. Relationships between the mycorrhizal and actinorhizal symbioses in nonlegumes. Methods in Microbiology 2:417-432.

Chacón-Madrigal, E; Wanek, W; Hietz, P; Dullinger, S. 2018. Traits indicating a conservative resource strategy are weakly related to narrow range size in a group of Neotropical trees. Perspectives in Plant Ecology, Evolution and Systematics 32:30-37.

Chaves-Corea, R. 2021. Contenido foliar de nutrimentos en especies arbóreas latifoliadas de Costa Rica. Tesis M.Sc. San José, Costa Rica, Universidad de Costa Rica. $181 \mathrm{p}$.

Chenery, EM; Sporne K. 1976. A note on the evolutionary status of aluminium-accumulators among dicotyledons. New Phytologist 76:551-554.

Condit, R; Engelbrecht, BMJ; Delicia-Pino, D; Pérez, R; Turner, BL. 2013. Species distributions in response to individual soil nutrients and seasonal drought across a community of tropical trees. Proceedings of the National Academy of Sciences 110(13):5064-5068.

Condit, R; Pérez, R; Daguerre, N. 2011. Trees of Panama and Costa Rica. Panamá, Panamá, Princenton Field Guides Series. 494 p.

Corrales, M; Bertsch, F; Bejarano, JA. 2005. Los laboratorios de análisis de suelos y foliares en Costa Rica: Informe del comité de laboratorios de análisis de suelos, plantas y aguas. Agronomía Costarricense 29(3):125-135.

Davidson, R; Gagnon, D; Mauffette, Y; Hernández, H. 1998. Early survival, growth and foliar nutrients in native Ecuadorian trees planted on degraded volcanic soil. Forest Ecology and Management 105(1-3):1-19.

Di Stéfano, JF; Fournier, LA. 1998. Biomasa aérea, concentración de nutrimentos y daños en árboles 
de Vochysia guatemalensis en un monocultivo de cuatro años, Tabarcia, Costa Rica. Agronomía Costarricense 22(2):235-241.

Drechsel, P; Zech, W. 1991. Foliar nutrient levels of broadleaved tropical trees: a tabular review. Plant and Soil 131(1):29-46.

Drechsel, P; Zech, W. 1993. Mineral nutrition of tropical trees. In Pancel, L (ed.). Tropical Forestry Handbook, Springer Verlag, Berlin, Vol. 1. p. 515-567.

Driessche van den, R. 1974. Prediction of mineral nutrient status of trees by foliar analysis. The Botanical Review 40(3):347-394.

Escobar, ML; Ortiz, JL; López, FL. 1993. Diagnóstico de daños nutricionales en cuatro especies forestales empleadas en reforestación. Medellín, Colombia, INDERENA. 88 p.

Evans, J. 1979. The effects of leaf position and leaf age in foliar analysis of Gmelina arborea. Plant and Soil 52(4):547-552.

Fallas, JL. 2014. Respuesta a la fertilización de la teca (Tectona grandis L.f.) con NPK en ultisoles de la zona norte de Costa Rica. Tesis Lic. Cartago, Costa Rica, Instituto Tecnológico de Costa Rica. 72 p.

Fernández-Moya, J. 2014. Gestión de la fertilidad de suelos y la nutrición de plantaciones de teca (Tectona grandis Lf) en América Central. Tesis Ph.D. Madrid, España, Universidad Politécnica de Madrid. 259 p.

Fernández-Moya, J; Alvarado, A. 2012. Nutrición y fertilización de Terminalia amazonia. Capítulo 19. In Alvarado, A; Raigosa, J (eds.). Nutrición y fertilización forestal en regiones Tropicales. San José, Costa Rica, Asociación Costarricense de la Ciencia del Suelo. p. 341-351.

Fernández-Moya, J; Murillo, R; Portuguez, E; Fallas, JL; Ríos, V; Kottman, F; Alvarado, A. 2013. Nutrient concentration age dynamics of teak (Tectona grandis Lf) plantations in Central America. Forest Systems 22(1):123-133.

Fernando, DR; Guymer, G; Reeves, RD; Woodrow, IE; Baker, AJM; Batianoff, GN. 2009. Foliar Mn accumulation in eastern Australian herbarium specimens: prospecting for 'new' Mn hyperaccumulators and potential applications in taxonomy. Annals of Botany 103(6):931-939.

Fisher, RF. 1995. Amelioration of degraded rain forest soils by plantations of native trees. Soil Science Society of America Journal 59(2):544-549.

Fonseca, W; Alice, FE; Rojas, JM; Villalobos, R; Porras, J; Spínola, M. 2016. Construcción de funciones alométricas para Costa Rica en el contexto del Proyecto de Protección Ambiental a través de la Protección de los Bosques de Centro América (Informe final), Costa Rica. 29 p.

Geoghegan, IE; Sprent, JI. 1996. Aluminum and nutrient concentrations in species native to central Brazil.
Communications in Soil Science and Plant Analysis 27(18-20):2925-2934.

Gómez, LD. 1986. Vegetación de Costa Rica. San José, Costa Rica, Editorial de la Universidad Estatal a Distancia (EUNED). 327 p.

González, E. 1996. Tropical tree species for reforestation: studies on seed storage, foliar nutrient content and wood variation. Tesis Ph.D. Texas, USA, A\&M Univ., College Station. 124 p.

González, E; Fisher, RF. 1997. Variación en la composición elemental foliar en árboles silvestres maduros y entre familias y procedencias de Vochysia guatemalensis en Costa Rica. Silvae Genetica 46(1):45-50.

Greaves, A; McCarter, PS. 1990. Cordia alliodora a promising tree for tropical agroforestry: Tropical Forestry Papers 22. Oxford Forestry Institute. University of Oxford. 37 p.

Guevara, G. 1988. Experiencias colombianas con cedro (Cedrela odorata L.). Corporación Nacional de Investigación y Fomento Forestal (CONIF). Serie de Documentación, $\mathrm{N}^{\circ}$ 12, Bogotá, Colombia. 86 p.

Haggar, J; Wightman, K; Fisher, RF. 1997. The potential of plantations to foster woody regeneration within a deforested landscape in lowland Costa Rica. Forest Ecology and Management 99(1-2):55-64.

Haridasan, M. 1982. Aluminium accumulation by some Cerrado native species of central Brazil. Plant and Soil 65(2):265-273.

Herrera, B. 1996. Evaluación del efecto del sitio en la productividad de las poblaciones de dos especies dominantes en un bosque tropical de la tercera fase de la sucesión secundaria en Costa Rica. Tesis M.Sc. Turrialba, Costa Rica, CATIE. 152 p.

Herrera, B; Finegan, B. 1997. Substrate conditions, foliar nutrients and the distributions of two canopy tree species in a Costa Rican secondary rain forest. Plant and Soil 191(2):259-267.

Horn, N; Montagnini, F. 1999. Litterfall, litter decomposition and maize bioassay of mulches from four indigenous tree species in mixed and monospecific plantations in Costa Rica. International Tree Crops Journal 10(1):37-50.

Jiménez, Q; Carrillo, E; Kappelle, M. 2015. The northern pacific lowland seasonal dry forest of Guanacaste and the Nicoya Peninsula. Costa Rican. Ecosystems. p. 247-289.

Johnson, P; Morales, R. 1972. A review of Cordia alliodora (Ruiz \& Pav.) Oken. Turrialba 22(2):210-220.

Kalra, Y. 1998. Handbook of reference methods for plant analysis. Soil and Plant Analysis Council, Inc. Boca Raton, FLA, CRC Press. 300 p.

Kitayama, K; Aiba, SI. 2002. Ecosystem structure and productivity of Tropical rain forests along altitudinal gradients with contrasting soil phosphorus pools on Mount Kinabalu, Borneo. Journal of Ecology 90(1):37-51. 
Klinge, H. 1985. Foliar nutrient levels of native tree species from Central Amazonia. 2. Campina. Amazonia 9(3):281-295.

Klinge, H; Furch, K; Harms, E; Revilla, J. 1983. Foliar nutrient levels of native tree species from Central Amazonia. 1. Inundation forests. Amazonia 8(1):19-45.

Kumar, JN; Kumar, RN; Kumar, BR; Sajish, PR. 2009. Quantification of nutrient content in the aboveground biomass of teak plantation in a Tropical dry deciduous forest of Udaipur, India. Journal of Forest Science 55(6):251-256.

Lavender, DP. 1970. Foliar analysis and how is used: a review, Research Note 52, Paper 691, Corvallis, Oregon, School of Forestry, Oregon State University. 8 p.

Lehto, T; Ruuhola, T; Dell, B. 2010. Boron in forest trees and forest ecosystems. Forest Ecology and Management 260(12):2053-2069.

Leitenmaier, B; Küpper, H. 2013. Compartmentation and complexation of metals in hyperaccumulator plants. Frontiers in Plant Science 4(374):1-13.

Leiva, JA. 2007. Regeneración arbórea y características edáficas en bosques secos tropicales desarrollados sobre la Meseta Ignimbrítica de Santa Rosa, Noroeste de Costa Rica. Tesis M.Sc. San José, Costa Rica, Universidad de Costa Rica. 148 p.

Leyton, L. 1948. Mineral nutrient relationships of forest trees. Forestry. Abstr.9: p. 399-408.

Lugo, AE; Martínez, OA; da Silva, JF. 2012. Aboveground biomass, wood volume, nutrient stocks and leaf litter in novel forests compared to native forests and tree plantations in Puerto Rico. Bois et Forets des Tropiques 314(4):7-16.

Ma, JF; Ueno, D; Zhao, FJ; McGrath, SP. 2005. Subcellular localization of $\mathrm{Cd}$ and $\mathrm{Zn}$ in the leaves of a Cd-hyperaccumulating ecotype of Thlaspi caerulescens. Planta 220(5):731-736.

Mills, HA; Jones, JB. 1996. Plant analysis handbook II (revised). A practical sampling, preparation, analysis, and interpretation guide. $\mathrm{N}^{\circ}$. 581.13 M657. Micromacro Publisher, Athens. 422 p.

Mitchell, HL. 1934. Pot culture tests of forest soil fertility with observations on the effect of varied solar radiation and nutrient supply on the growth and nitrogen content of Scots and White Pine seedlings. Black Rock Forest, Bulletin s: 1-138. NY, USA, Cornwall-on-the-Hudson. s.p.

Montagnini, F. 2000. Accumulation in above-ground biomass and soil storage of mineral nutrients in pure and mixed plantations in a humid Tropical lowland. Forest Ecology and Management 134(1-3):257-270.

Montagnini, F; Sancho, F; González, E; Moulaert, A. 1993. Mixed-tree plantations with indigenous trees in the Atlantic lowlands of Costa Rica. In Management and rehabilitation of degraded lands and secondary forests in Amazonia. Proceedings of an International
Symposium. Santarem, Pará, Brazil. IITF-USDAForest Service, Puerto Rico. p 161-169.

Montenegro, E. 2005. Efecto del aporte de nutrientes de la biomasa de tres tipos de árboles de sombra en sistemas de manejo de café orgánico y convencional Tesis M.Sc. Turrialba, Costa Rica, CATIE. 67 p.

Montero, M. 1999. Factores de sitio que influyen en el crecimiento de Tectona grandis L.f. y Bombacopsis quinata (Jacq.) Dugand, en Costa Rica. Tesis M.Sc. Valdivia, Chile, Universidad Austral de Chile. 111 p.

Murillo, RA. 1996. Evaluación de algunos factores ambientales que afectan la calidad de sitio a nivel de micrositio para melina (Gmelina arborea Roxb. plantada en suelos planos en la zona sur de Costa Rica. Tesis Lic. Heredia, Costa Rica, Universidad Nacional. 111 p.

Murillo, RA. 2013. Contenido de nutrimentos en la biomasa aérea de árboles de teca (Tectona grandis L.f.) en la cuenca del Canal de Panamá. Tesis M.Sc. San José, Costa Rica, Universidad de Costa Rica. 126 p.

Murillo, RA; Alvarado, A. 2012. Nutrición y fertilización de Gmelina arborea. Capítulo 15. In Alvarado A, Raigosa, J (eds.). Nutrición y fertilización forestal en regiones Tropicales. San José, Costa Rica, Asociación Costarricense de la Ciencia del Suelo. p. 281-294.

Murillo, RA; Alvarado, A; Verjans, JM. 2014. Concentración foliar de nutrimientos en plantaciones de teca en la cuenca del Canal de Panamá. Agronomía Costarricense 38(1):11-28.

Murphy, PG; Lugo, AE. 1995. Dry forests of Central America and the Caribbean, Seasonally Dry Tropical Forest. p. 9-34.

Negi, MS; Tandon, YN; Rawat, HS. 1995. Biomass and nutrient distribution in young teak (Tectona grandis Linn. f) plantations in Tarai region of Uttar Pradesh. Indian Forester 121(6):455-464.

Nichols, JD; Gillespie, AR; Richter, DD. 1997. Growth, foliar and nutrient status of Terminalia amazonia planted in southwestern Costa Rica. Journal of Tropical Forest Science 10:233-248.

Nwoboshi, LC. 1975. Macronutrient deficiency symptoms in teak (Tectona grandis L.f.). Bulletin $\mathrm{N}^{\circ}$. 6 . Department of Forest Research Management, University of Ibadan. $12 \mathrm{p}$.

Nwoboshi, LC. 1984. Growth and nutrient requirements in a teak plantation age series in Nigeria. II. Nutrient accumulation and minimum annual requirements. Forest Science 30(1):35-40.

Onweremadu, EU. 2007. Pedogenic loss and uptake of calcium by Gmelina growing in an isohyperthermic Kandiudult. Plant Science 2:625-629.

Ordóñez, H. 2003. Fenología de la copa y de las raíces finas de Simarouba glauca y Dalbergia retusa (cocobolo) con riego en una plantación mixta de Guanacaste. 
Tesis M.Sc. San José, Costa Rica, Universidad de Costa Rica. 134 p.

Pancel, L. 2015. Species files in tropical forestry. In Pancel, L; Köhl, M (eds.). Tropical Forestry Handbook, vol. $\mathrm{N}^{o}$ 2. Berlin, Heidelberg, Springer-Verlag. p. 1203-1220

Paudyal, BK. 2012. Forest nutrition research and its role in forest management. Institute of Forestry, Tribhuvan University Pokhara Campus, Pokahra. 223 p.

Peck, RB. 1976. Selección preliminar de especies aptas para el establecimiento de bosques artificiales en tierra firme del litoral pacífico de Colombia. Boletín del Instituto Forestal Latinoamericano de Investigación y Capacitación (50):29-39.

Pérez, J; Bornemisza, E; Sollins, P. 1993. Identificación de especies forestales acumuladoras de aluminio en una plantación forestal experimental ubicada en Sarapiquí, Costa Rica. Agronomía Costarricense 17(2):99-103.

Portuguez, EM. 2012. Estimación de la extracción de nutrimentos por parte aérea en plantaciones de teca (Tectona grandis Linn. f.) de las empresas Panamerican Woods y C\&M Investment Group Ltda. en la Península de Nicoya, Guanacaste, Costa Rica. Tesis Lic. San José, Costa Rica, Universidad de Costa Rica. 92 p.

Pregitzer, KS; King, JS. 2005. Effects of soil temperature on nutrient uptake. In Nutrient Acquisition by Plants. Berlin, Heidelberg, Springer. p. 277-310.

Ramírez, D. 2014. Absorción de nutrimentos de cedro amargo (Cedrela odorata L.) como mecanismo de diagnóstico para mejorar el manejo de la fertilización de la especie para agricultores asociados a COOPEAGRI R.L. Tesis Lic. San José, Costa Rica, Universidad de Costa Rica. 81 p.

Rascio, N; Navari-Izzo, F. 2011. Heavy metal hyperaccumulating plants: how and why do they do it? And what makes them so interesting? Plant Science 180(2):169-181.

Raulino, WNC; Freire, FJ; Assunção, EADA; Ataide, KMPD; Silva, HVD; Silva, ACFD. 2020. Nutrition of tree species in tropical dry forest and rainforest environments. Revista Ceres 67(1):70-80.

Reyes, CE. 1997. Estimación del incremento diamétrico en Cordia alliodora y Vochysia ferruginea a partir de variables del árbol y factores del sitio en un bosque secundario en Costa Rica. Tesis M.Sc. Turrialba, Costa Rica, CATIE. $134 \mathrm{p}$

Rodríguez-Barrueco, C; Miguel, C; Subramaniam, P. 1984. Seasonal fluctuations of the mineral concentration of alder (Alnus glutinosa (L.) Gaertn.) from the field. Plant and Soil 78(1-2):201-208.

Rojas, F; Murillo, O. 2004. Botánica y ecología. Manual para productores de melina (Gmelina arborea) en Costa Rica, Cartago, Costa Rica. p. 3-21.
Sampaio, L; Huber, W. 1999. Concentração e distribuição de nutrientes nas folhas de espécies florestais, na Amazônia Ocidental. Floresta e Ambiente 6(1):127-137.

Segura, JM. 2005. Respuesta del jaúl (Alnus acuminata ssp. arguta) a la fertilización fosfórica en Andisoles del cantón de Coronado, Costa Rica. Tesis M.Sc. Universidad de Costa Rica, San José, Costa Rica. 151 p.

Segura, JM; Castillo, Á; Alvarado, A; Blanco, F. 2006. Variación del contenido foliar de nutrimentos de Alnus acuminata. Agronomía Costarricense 30(1):53-63.

Sharma, E. 1993. Nutrient dynamics in Himalayan alder plantations. Annals of Botany 72(4):329-336.

Siddiqui, M; Shah, A; Yaqoob, S. 2009. Chronosequence and crown strata effects on foliar nutrient concentrations in teak (Tectona grandis Lf) plantations. Pakistan Journal of Botany 41:3023-3034.

Smith, FW; Loneragan, JF. 1997. Interpretation of plant analysis: concepts and principles. In Reuter, DJ; Robinson, JB (eds.). Plant Analysis and Interpretation Manual, Australia, CSIRO Publishing. p. 35-51.

Soethe, N; Lehmann, J; Engels, C. 2008. Nutrient availability at different altitudes in a tropical montane forest in Ecuador. Journal of Tropical Ecology 24(4):397-406.

Stuhrmann, M; Bergmann, C; Zech, W. 1994. Mineral nutrition, soil factors and growth rates of Gmelina arborea plantations in the humid lowlands of northern Costa Rica. Forest Ecology and Management 70(1-3):135-145.

Tanner, EVJ; Vitousek, PA; Cuevas, E. 1998. Experimental investigation of nutrient limitation of forest growth on wet tropical mountains. Ecology 79(1):10-22.

Vaides, EE. 2004. Características de sitio que determinan el crecimiento y productividad de teca (Tectona grandis $\mathrm{Lf}$ ), en plantaciones forestales de diferentes regiones en Guatemala. Tesis M.Sc. Turrialba, Costa Rica, CATIE. 68 p.

Vallejos, OS. 1996. Productividad y relaciones del índice de sitio con variables fisiográficas, edafoclimáticas y foliares para Tectona grandis L.f., Bombacopsis quinata (Jacq) Dugand y Gmelina arborea Roxb. en Costa Rica. Tesis M.Sc. Turrialba, Costa Rica, CATIE. 147 p.

Van der Ent, A; Baker, AJM; Reeves, RD; Pollard, AJ; Schat, H. 2013. Hyperaccumulators of metal and metalloid trace elements: facts and fiction. Plant and Soil 362(1-2):319-334.

Vásquez, W; Ugalde, LA. 1995. Rendimiento y calidad de sitio para Gmelina arborea, Tectona grandis, Bombacopsis quinata y Pinus caribaea, en Guanacaste, Costa Rica. CATIE/ IDA/ FAO/ HOLANDA. Proyecto Madeleña-3. Turrialba, Costa Rica. Serie Técnica, Informe Técnico, No. 256. 33 p. 
Webb, MJ; Reddell, P; Hambleton, A; Robson, KEN. 2000. Growth response of four tropical plantation timber species to increasing phosphorus supply and assessment of phosphorus requirements using foliar analysis. New Forests 20(2):193-211.
Zech, W; Drechsel, P. 1992. Multiple mineral deficiencies in forest plantations in Liberia. Forest Ecology and Management 48(1-2):121-143.

\footnotetext{
(C) $\$\left(\begin{array}{l}\text { BY NC ND } \\ \text { Todos los derechos reservados. Universidad de Costa Rica. Este artículo se encuentra licenciado con Creative Commons } \\ \text { Reconocimiento-NoComercial-SinObraDerivada } 3.0 \text { Costa Rica. Para mayor información escribir a rac.cia@ ucr.ac.cr }\end{array}\right.$
} 
